Provided for non-commercial research and education use. Not for reproduction, distribution or commercial use.

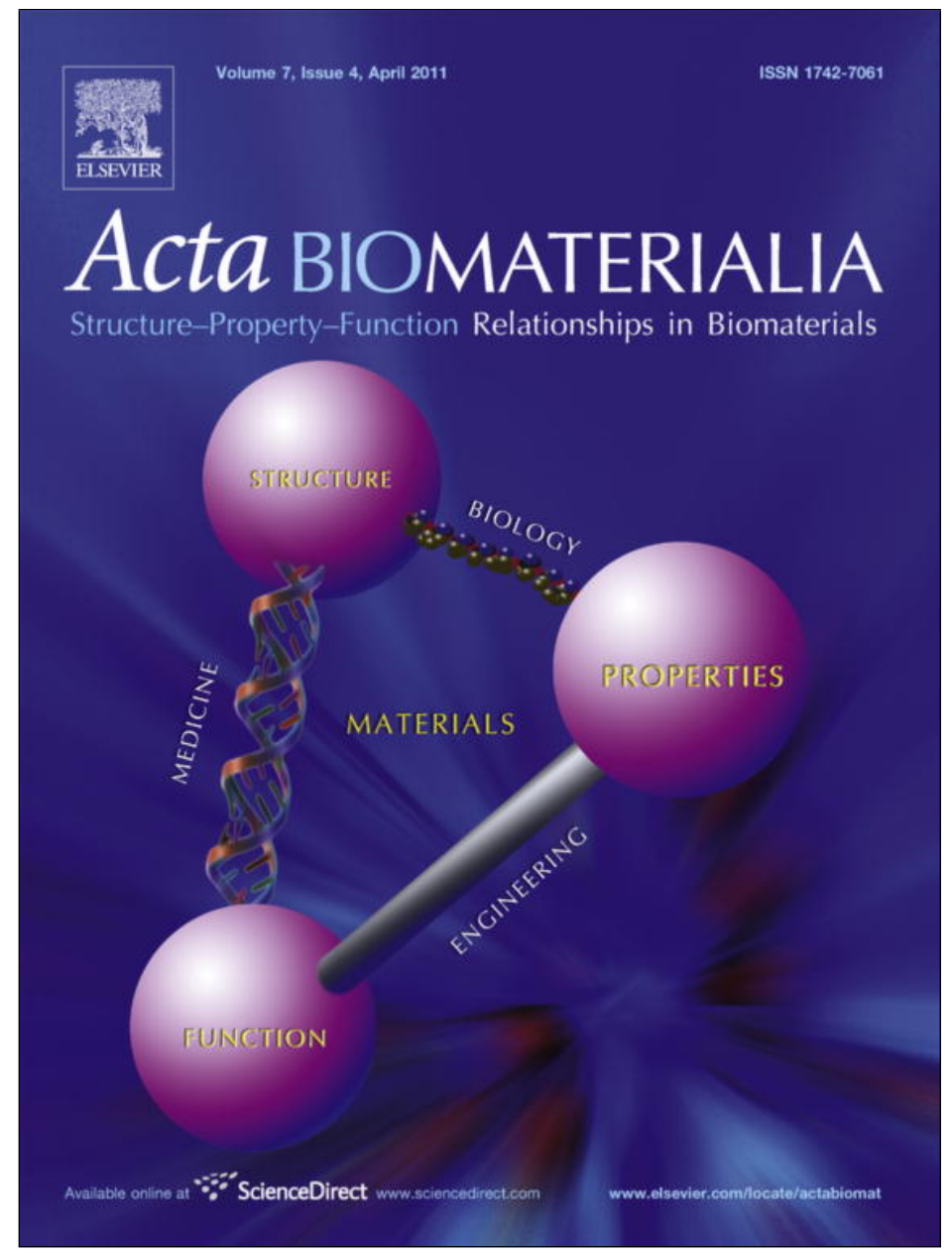

This article appeared in a journal published by Elsevier. The attached copy is furnished to the author for internal non-commercial research and education use, including for instruction at the authors institution and sharing with colleagues.

Other uses, including reproduction and distribution, or selling or licensing copies, or posting to personal, institutional or third party websites are prohibited.

In most cases authors are permitted to post their version of the article (e.g. in Word or Tex form) to their personal website or institutional repository. Authors requiring further information regarding Elsevier's archiving and manuscript policies are encouraged to visit:

http://www.elsevier.com/copyright 


\title{
Effect of flow perfusion conditions in the chondrogenic differentiation of bone marrow stromal cells cultured onto starch based biodegradable scaffolds
}

\author{
Alexandra Gonçalves ${ }^{\mathrm{a}, \mathrm{b}}$, Pedro Costa ${ }^{\mathrm{a}, \mathrm{b}}$, Márcia T. Rodrigues ${ }^{\mathrm{a}, \mathrm{b}}$, Isabel R. Dias ${ }^{\mathrm{a}, \mathrm{b}, \mathrm{c}}$, Rui L. Reis ${ }^{\mathrm{a}, \mathrm{b}}$, \\ Manuela E. Gomes ${ }^{\mathrm{a}, \mathrm{b}, *}$ \\ a 3B's Research Group-Biomaterials Biodegradables and Biomimetics, Department of Polymer Engineering, University of Minho, \\ Headquarters of the European Institute of Excellence on Tissue Engineering and Regenerative Medicine, AvePark, 4806-909 Taipas, Guimarães, Portugal \\ ${ }^{\mathrm{b}}$ IBB-Institute for Biotechnology and Bioengineering, PT Government Associated Laboratory, Guimarães, Portugal \\ ${ }^{\mathrm{c}}$ Department of Veterinary Sciences, School of Agrarian and Veterinary Sciences, University of Trás-os-Montes e Alto Douro, P.O. Box 1013, 5000-911 Vila Real, Portugal
}

\section{A R T I C L E I N F O}

\section{Article history:}

Received 18 August 2010

Received in revised form 28 October 2010

Accepted 30 November 2010

Available online 3 December 2010

\section{Keywords:}

GBMCs

SPCL

Flow perfusion system

Cartilage

Chondrogenic differentiation

\begin{abstract}
A B S T R A C T
Cartilage tissue engineering (TE) typically involves the combination of a 3-D biodegradable polymeric support material, with primary chondrocytes or other cell types able to differentiate into chondrocytes. The culture environment in which cell-material constructs are created and stored is an important factor. Various bioreactors have been introduced in TE approaches to provide specific culturing environments that might promote and accelerate cells' potential for chondrogenic differentiation and enhance the production of cartilage extracellular matrix. The aim of the present study was to investigate the chondrogenic differentiation of goat bone marrow cells (GBMCs) under flow perfusion culture conditions. For that purpose, GBMCs were seeded into starch-polycaprolactone fiber mesh scaffolds and cultured in a flow perfusion bioreactor for up to 28 days using culture medium supplemented with transforming growth factor- $\beta 1$. The tissue-engineered constructs were characterized after several end points $(7,14$, 21 and 28 days) by histological staining and immunocytochemistry analysis, as well as by glycosaminoglycan and alkaline phosphatase quantification assays. In addition, the expression of typical chondrogenic markers was assessed by real-time reverse-transcription polymerase chain reaction analysis. In general, the results obtained suggest that a flow perfusion microenvironment favors the chondrogenic potential of GBMCs.
\end{abstract}

(c) 2010 Published by Elsevier Ltd. on behalf of Acta Materialia Inc.

\section{Introduction}

Hyaline cartilage is an avascular, aneural and alymphatic, but highly specialized, type of connective tissue [1-3]. Cartilage is composed by chondrocytes that produce a large amount of extracellular matrix (ECM), made of collagen fibrils, predominantly type II collagen and link proteins such as proteoglycans (PGs), aggrecan and hyaluronic acid [4-6]. Cartilage, found within many joints, is responsible for the correct functionality of the articulating skeleton, creating smooth gliding areas in the terminal parts of long bones for shock absorbance, load bearing and reduction of surface friction $[1,2]$.

Damaged articular cartilage shows a limited capacity for selfregeneration $[1,5]$ due to the low metabolic activity and prolifera-

\footnotetext{
* Corresponding author at: 3B's Research Group-Biomaterials Biodegradables and Biomimetics, Department of Polymer Engineering, University of Minho, Headquarters of the European Institute of Excellence on Tissue Engineering and Regenerative Medicine, AvePark, 4806-909 Taipas, Guimarães, Portugal. Tel.: +351 253510 906; fax: +351253510909.

E-mail address: megomes@dep.uminho.pt (M.E. Gomes).
}

tion rates of mature chondrocytes, and because of the absence of either vascular or lymphatic vessels $[2,4,6]$. These limitations led to the development of surgical methods for restoring cartilage surface; however, to date, none of these procedures has been able to reliably reproduce hyaline cartilage.

Cartilage tissue engineering (TE) appears to be a promising approach for improving the quality and longevity of repaired tissue [7]. The general principle of cartilage TE involves a natural/synthetic biodegradable material or scaffold combined with chondrocytes or mesenchymal stromal cells to build a three dimensional (3-D) living construct functionally, structurally and mechanically similar to the tissue to be replaced [5]. Several combinations of materials processed into 3-D porous scaffolds or hydrogels, and various cell types have been used in cartilage TE strategies [8]. Mesenchymal stromal cells (MSCs), due to their ability to proliferate extensively ex vivo, while maintaining multipotent differentiation properties, are an attractive cellular source for cell-based cartilage repair strategies [6,9-12]. In vitro chondrogenic differentiation of MSCs involves complex interfaces of growth factors, cellcell and cell-matrix interactions. Many studies have demonstrated the chondrogenic differentiation of MSCs in the presence of the 
transforming growth factor- $\beta$ family $[8,11]$, whose members are known to induce the expression of cartilage-specific genes and ECM deposition [2].

Bioreactor studies have focused on the influence of mechanical stimuli on the proliferation and differentiation of MSCs [11,13]. Therefore, the design of bioreactors constitutes another important element for TE strategies, as these culturing systems provide optimized environments for in vitro functional 3-D tissue development [14-16]. Problems associated with poor diffusion under traditionally static culturing conditions can be mitigated using a flow perfusion bioreactor, in which media is forced into the scaffold pores $[9,14,17]$. A fluid flow culture system not only provides more efficient nutrient supply and metabolite removal, but also furnishes a physiochemical environment favorable for stimulating undifferentiated cells into target phenotypes [13,17].

The present work investigates the influence of cell culture conditions generated by a flow perfusion bioreactor system on the proliferation and chondrogenic differentiation of goat bone marrow stromal cells (GBMCs) seeded onto starch-polycaprolactone (SPCL) scaffolds.

\section{Materials and methods}

\subsection{Preparation of biodegradable supports-SPCL fiber mesh scaffold}

Fiber-mesh scaffolds based on SPCL (a blend of corn starch and polycaprolactone, 30/70 wt.\%) were prepared by fiber bonding, a technique that consists of cutting and sintering fibers previously obtained by melt-spinning [18]. Scaffolds were cut into samples $8 \mathrm{~mm}$ in diameter and 3.5-4 $\mathrm{mm}$ high, and then sterilized with ethylene oxide.

\subsection{Goat bone marrow stromal cell culture and chondrogenic differentiation}

GBMCs were harvested from the iliac crest of adult goats and expanded until passage 3 (P3) in low-glucose Dulbecco's modified essential medium (DMEM; Sigma Chemical Co., St. Louis, MO, USA) supplemented with $1 \%$ antibiotic/antimycotic (Sigma) and $10 \%$ fetal bovine serum (FBS; Sigma) [1]. The cells used in this study were harvested from the same animal, as GBMCs had previously been expanded and cryopreserved under the same conditions. The only difference is that different vials of the same lot of cells were thawed and used in the pellet and SPCL scaffold studies.

\subsubsection{Pellet culture system}

The potential of GBMCs to undergo chondrogenic differentiation was assessed by culturing cell pellets. For the preparation of each pellet, aliquots of $2.5 \times 10^{5}$ cells in $0.5 \mathrm{ml}$ of medium were centrifuged at $1500 \mathrm{rpm}$ for $15 \mathrm{~min}$ in a $15 \mathrm{ml}$ polypropylene conical tube. Pellets were then cultured for 28 days in a $37^{\circ} \mathrm{C}$ humidified atmosphere including $5 \% \mathrm{CO}_{2}$; the medium was changed once a week. The medium used for chondrogenic differentiation was composed by low-glucose DMEM (Sigma), 1\% antibiotic/antimicotic (Sigma), and 5\% FBS supplemented with $10 \%$ Insulin-Transferrin-Selenium (ITS(+); Sigma), $17 \mathrm{mM} \mathrm{L-ascorbic} \mathrm{acid} \mathrm{(Sigma),}$ $0.1 \mathrm{M}$ sodium pyruvate (Sigma), $35 \mathrm{mM}$ L-proline (Sigma), $1 \mathrm{mM}$ dexamethasone (Sigma) and $10 \mathrm{ng} \mathrm{ml}^{-1}$ TGF- $\beta 1$ (Sigma-Aldrich, Portugal).

\subsubsection{Cell seeding onto SPCL scaffolds}

After reaching confluence, GBMCs were detached using trypsin/ EDTA (0.05\% w/v trypsin/0.02\% EDTA; Sigma). Cells (P3) were then centrifuged at $1200 \mathrm{rpm}$ for $5 \mathrm{~min}$ and resuspended in basal medium. Subsequently, SPCL scaffolds were placed in non-adherent 48- well plates and seeded with a $150 \mu$ cell suspension containing $5 \times 10^{5}$ cells, followed by $2 \mathrm{~h}$ incubation. Afterwards, $500 \mu \mathrm{l}$ of basal medium was added to each well, and incubated overnight to enable cell attachment. On the following day, samples were transferred to other well-plates and kept either in static conditions or moved into the bioreactor system.

\subsubsection{Cell culture: flow perfusion bioreactor}

The flow perfusion bioreactor used in this study was developed by the 3B's Research Group [19,20]. The system is composed of 20 culture chambers, each one supporting a single, tightly fitting scaffold. A peristaltic pump controls the medium flow rate through the bioreactor, with silicon tubes directly connected to the system and to a medium reservoir. In this experiment, a continuous flow rate of $0.1 \mathrm{ml} \mathrm{min}^{-1}$ was induced for each scaffold, keeping the total volume in the flow system at $100 \mathrm{ml}$. The chondrogenic medium was then added to constructs from both culturing conditions (static and flow perfusion), and medium changed once a week. $0.5 \mathrm{ml}$ medium was also added to each cell-SPCL construct kept under static culture. Control constructs were cultured in DMEM. All GBMC-SPCL constructs were maintained in a $37^{\circ} \mathrm{C}$ environment with $5 \% \mathrm{CO}_{2}$. SPCL scaffolds without cells were also kept under the same conditions and used as negative controls.

\subsection{Characterization of pellets and cell-scaffold constructs}

\subsubsection{Cell metabolic activity}

Cellular metabolic activity was assessed by MTS assay. Culture medium was removed and samples were rinsed in PBS. Afterwards, MTS reagent (VWR, Portugal) was added, and incubated for $3 \mathrm{~h}$. MTS precipitate, produced by metabolically active cells in culture, was quantified by spectrophotometry at $\mathrm{A}_{490} \mathrm{~nm}$, using a microplate reader (Bio-Tek, Synergie HT, USA).

\subsubsection{Cell proliferation}

To estimate the cellular proliferation rate during the culture time, double-stranded DNA (dsDNA) content was measured using a fluorimetric PicoGreen dsDNA Quantification Kit (P7589, Invitrogen, Molecular Probes, USA). For this purpose, samples collected after each end point were carefully rinsed in PBS, and kept in $1 \mathrm{ml}$ ultrapure water at $-80^{\circ} \mathrm{C}$ until usage. Prior to DNA quantification, samples were thawed and sonicated for $15 \mathrm{~min}$. Experimental samples and standards $\left(0-2 \mu \mathrm{g} \mathrm{ml}^{-1}\right)$ were added to a white opaque 96 -well plate. The followed procedure was based on the manufacturer instructions. Fluorescence was quantified using a microplate reader described above at an excitation of $A_{485 / 20}$ and at an emission of $A_{528 / 20}$.

\subsubsection{Alkaline phosphatase quantification}

Alkaline phosphatase (ALP) activity was measured using a colorimetric endpoint assay which quantified the conversion of $p$ nitrophenol phosphate to $p$-nitrophenol by ALP enzyme. Briefly, samples and a substrate solution of $0.2 \%(\mathrm{w} / \mathrm{v}) \mathrm{p}$-nitrophenyl phosphate (Sigma) in a substrate buffer of $1 \mathrm{M}$ diethanolamine $\mathrm{HCl}$ (Merck, Germany) were added to a 96-well plate. After $1 \mathrm{~h}$ of incubation, a stop solution (2 $\mathrm{M} \mathrm{NaOH}$ (Panreac) plus $0.2 \mathrm{mM}$ EDTA (Sigma)), was added to each well, and the absorbance read at $405 \mathrm{~nm}$ in a microplate reader. A standard curve was made from standards $\left(0-0.3 \mu \mathrm{mol} \mathrm{ml}{ }^{-1}\right)$ prepared with a $p$-nitrophenol (pNP; Sigma) solution. Sample and standard triplicates were analyzed and sample concentrations read off from the standard curve.

\subsubsection{Glycosaminoglycan content}

The presence of PGs was determined by measuring the level of sulfate glycosaminoglycans (GAGs) using a 1,9-dimethylmethylene blue (DMB; Aldrich) dye-binding assay and quantified using a 
6-sulfate chondroitin standard curve. Samples were digested in a sodium phosphate-buffered protein $\mathrm{k}$ (Sigma) solution at $60^{\circ} \mathrm{C}$ overnight. After the digestion was completed, samples and chondroitin sulfate standards were placed in a 96-well plate followed by a DMB metachromatic solution. The optical density was measured using a microplate reader at $A_{520 / 530} \mathrm{~nm}$. A standard curve was created and GAG values of the samples were read off from the standard graph.

\subsubsection{Scanning electron microscopy}

GBMC-SPCL interactions, cellular adhesion and proliferation were observed by scanning electron microscopy (SEM). Representative samples of each culture condition at each end point $(7,14$ 21 and 28 days) were rinsed in PBS and fixed in a solution of $2.5 \%$ glutaraldehyde (in PBS). Before sputter coating with gold, samples were dehydrated in a gradient series of ethanol solutions and air dried at room temperature. Samples were then observed using a scanning electron microscope (Leica Cambridge S360, Leica Cambridge, UK).

\subsubsection{Histological and immunocytochemistry analysis}

2.3.6.1. Pellet characterization. Pellets were rinsed in PBS, fixed in $4 \%$ formalin, embedded in paraffin and cut into $2.5 \mu \mathrm{m}$ sections. In order to observe cells and matrix formed, sections were identified with hematoxylin \& eosin (H\&E; Sigma). PGs and GAGs were stained with Alcian blue (1\%, Sigma), Toluidine blue (1\%, Sigma) and Safranin $\mathrm{O}(0.1 \%$, Fluka), which are frequently used as cartilage-specific stainings. Type IIA collagen was detected by immunocytochemistry using a RTU Vectastain Universal Elite ABC Kit Pk-7200 and a Vector DAB Kit (Vector Laboratories Ltd. UK). The procedure was based on the protocol provided by the manufacturer. Samples were then visualized under a light microscope (Stemi 1000 PG-HITEC Zeiss) and images taken with an attached camera (Axion MRc5, Zeiss).

2.3.6.2. GBMC-SPCL construct characterization. GBMC-SPCL constructs were rinsed in PBS and fixed with $4 \%$ formalin. Constructs were directly stained with Safranin O, and then visualized with a stereomicroscope (Stemi 2000 Zeiss) equipped with an imaging camera (PowerShot G6, Canon).

\subsubsection{Measurement of $m R N A$ level by real-time reverse-transcription} polymerase chain reaction

mRNA expression of the genes of interest was measured in the pellet culture systems as well as in the GBMC-SPCL constructs, both in static and dynamic culture conditions, by real-time reverse-transcription polymerase chain reaction (RT-PCR) analysis. Total RNA was extracted using TRIzol (Invitrogen, USA) following the manufacturer's instructions. First-strand complementary DNA (cDNA) was synthesized from $1 \mu \mathrm{g}$ RNA using the iscript cDNA synthesis Kit (BioRad, USA) in a $20 \mu \mathrm{l}$ reaction. The amount of cDNA was measured by quantitative PCR method using iQ SYBER Green SuperMix (VWR, Portugal), according to the manufacturer's instructions, in a $25 \mu \mathrm{l}$ reaction containing $5 \mu \mathrm{l}$ of primers. The primer sequence, with specificity for goat, was obtained from Primer3 software (v 0.4.0) for GAPDH, type II and type I collagen and Sox 9 genes, and synthesized by MWG Biotech Germany (see Table 1). Relative quantification was calculated using the $2^{-\Delta \mathrm{Ct}}$ method for pellets and $2^{-\Delta \Delta C t}$ method for GBMC-SPCL constructs [21]. mRNA expression levels of target genes were normalized to an endogenous housekeeping gene, GAPDH. Undifferentiated GBMCs seeded onto SPCL scaffolds cultured in basic medium with DMEM $(7,14$ 21 and 28 days) were used as calibrators.
Table 1

Goat chondrogenic primer pairs.

\begin{tabular}{|c|c|c|c|}
\hline Gene & Amplicon (bp) & Primers & ID number \\
\hline \multirow[t]{2}{*}{ GAPDH } & 176 & F-5' GGGTCATCATCTCTGCACCT 3' & AJ431207 \\
\hline & & R-5' GGTCATAAGTCCCTCCACGA 3' & \\
\hline \multirow[t]{2}{*}{ Col IIA } & 101 & F-5' CTGGATGCCATGAAGGTTTT 3' & FJ178374 \\
\hline & & R- 5' CTCTTGCTGCTCCACCAGTT 3' & \\
\hline \multirow[t]{2}{*}{ Sox 9} & 243 & F-5' TGAAGAAGGAGAGCGAGGAG $3^{\prime}$ & EF203710 \\
\hline & & R-5' TGCCCAGAGTCTTGCTGAG 3' & \\
\hline \multirow[t]{2}{*}{ Col I } & 167 & F-5' GGTGACAGGAAGTCCCAGAA 3' & FJ200436 \\
\hline & & R-5' CCATCGTAGGTGACGCTGTA 3' & \\
\hline
\end{tabular}

\subsection{Statistical analysis}

All values are presented as mean \pm standard deviations. For statistical analysis multiple-pairs comparison have been performed using one-way ANOVA followed by the Tukey-Kramer method with a significance level of $95 \%(P<0.05)$.

\section{Results}

\subsection{Pellet system culture}

In our experiment, the chondrogenic potential of the isolated GBMC population was studied and confirmed in vitro using pellet culture systems for up to 28 days. This is a 3-D cell culture technique designed to improve the differentiation of stromal cells into chondrocytes in the presence of a chondrogenic differentiation factor, TGF- $\beta 1$. Cell proliferation was evaluated at different end points (7, 14 and 28 days) (Fig. 1), showing a tendency to decrease with the time in culture.

The cellular metabolic activity showed a decrease from day 7 to day 14 , followed by an increase in cell viability from day 14 to day $28(P<0.05)$, indicating that low DNA values are not related to cell death, but are probably involved in the chondrogenic differentiation process (Fig. 2).

Examination of histological sections of pellets harvested after 7 and 28 days in culture and stained with H\&E showed no notable morphological differences with time (Fig. 3A, B). An intense color developed by Alcian blue, Toluidine blue and Safranin $O$ stainings indicated the presence of PGs and GAGs in the pellet structures (Fig. 3C-H), suggesting that cartilaginous matrix had been synthesized.

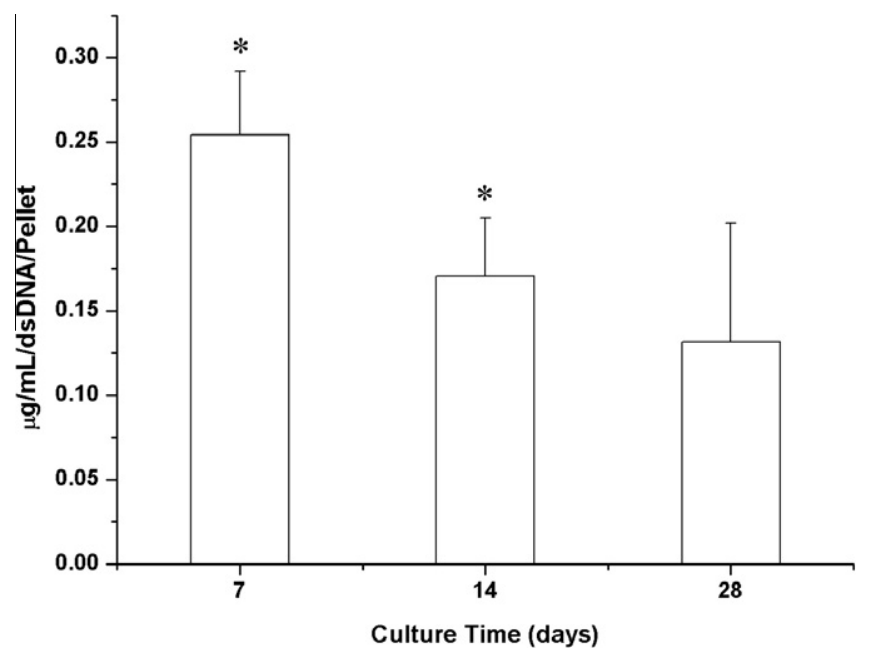

Fig. 1. DNA quantification of pellets harvested at different time periods of culture Results presented represent mean \pm SD. $*$ Statistically significant differences $(P<0.05)$. 


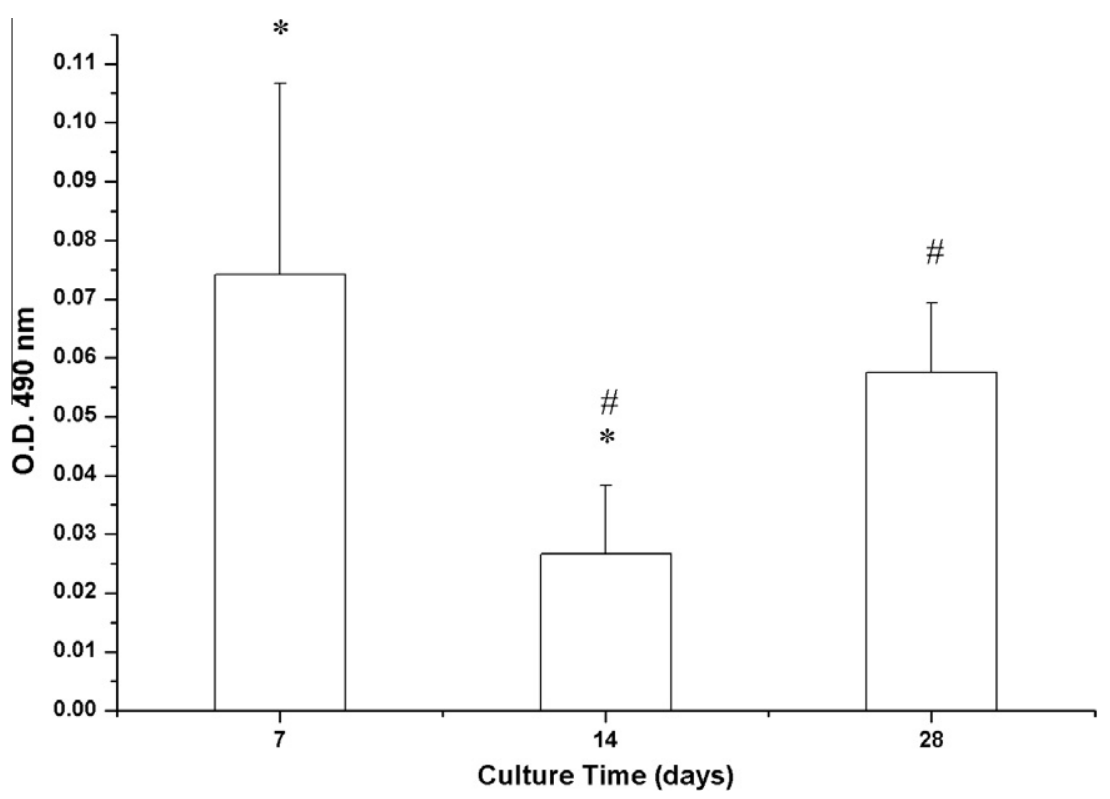

Fig. 2. Cell viability assay of GBMCs pellets using MTS reagent. Results presented represent mean \pm SD. $*$ Statistically significant differences $(P<0.05)$.

H\&E
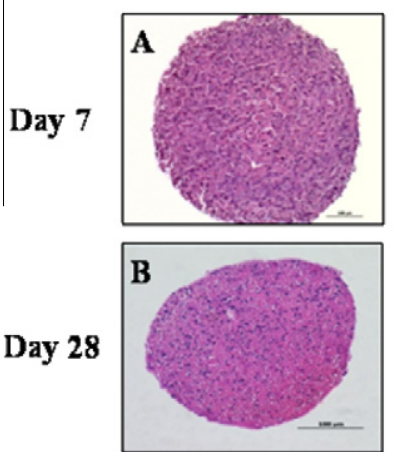

Alcian blue
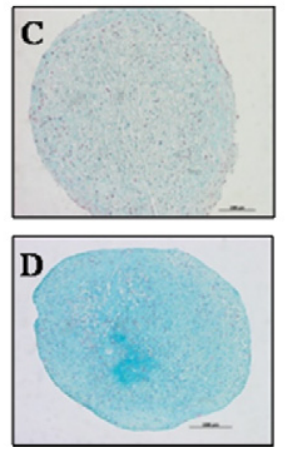

Toluidine blue
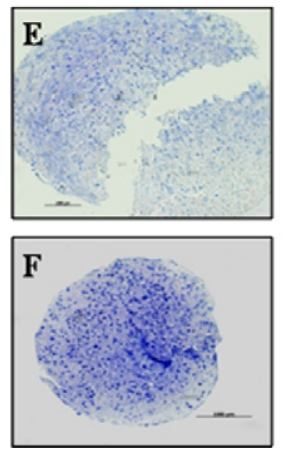

Safranin O
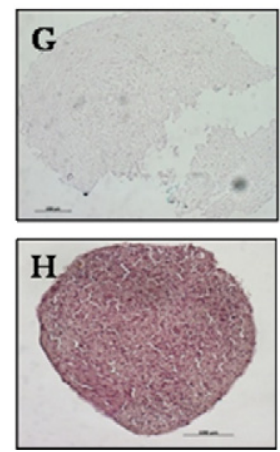

Fig. 3. Light microscopy images obtained from pellets sections after H\&E staining (A and B); Alcian blue staining (C and D); Toluidine blue (E and F) and Safranin O ( $G$ and $H$ ). Scale bar $=100 \mu \mathrm{m}$.

Day 7

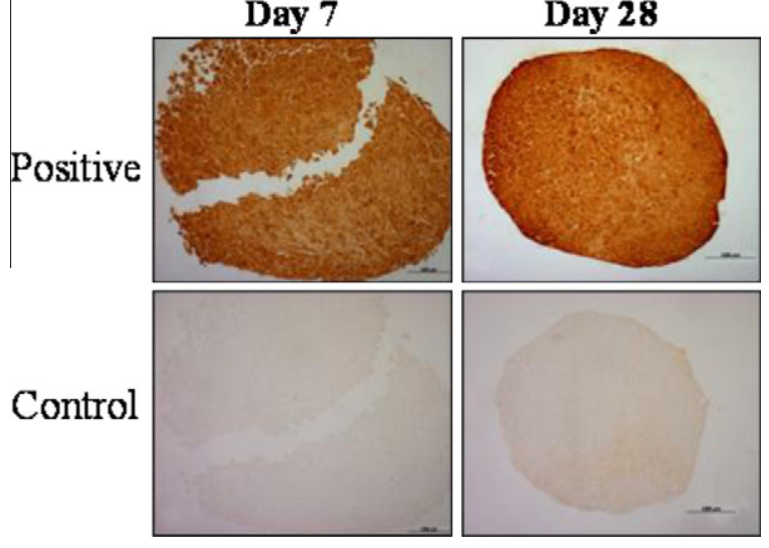

Fig. 4. Images obtained from pellets sections that were immunocytochemically stained for collagen type IIA after 7 and 28 days of culture in the presence of TGF- $\beta$ (positively stained and control section). Scale bar $=100 \mu \mathrm{m}$.

These findings were further supported by immunocytochemical results, which detected type IIA collagen, a major protein of cartilaginous ECM (Fig. 4) in pellets from every culture time studied.
Total mRNA was extracted from GBMCs pellets at different culture times. The mRNA of different genes was measured and normalized to the expression of housekeeping GAPDH. The mRNA expression of Sox9, type IIA and type IA collagen was detected in all pellets and some significant differences were found in some time points (Fig. 5). Despite the higher expression of Sox9 through time, type IIA collagen had expression values superior to type IA collagen (also strongly detected by immunocytochemical analysis).

\subsection{GBMC-SPCL constructs in flow perfusion culture}

Constructs harvested from the bioreactor and static cultures were characterized with respect to dsDNA content and cellular metabolism after $0,7,14,21$ and 28 days. Comparing the static culture with the flow perfusion system, differences in levels are evident both for cellular metabolism and proliferation rates (Fig. 6A and B). The GAG content (Fig. 6C) in the constructs seems to be higher when cells were cultured under static conditions. However, the GAG content increased up to 7 days, followed by maintenance levels under static and dynamic cultures. Constructs from both culture conditions exhibited low ALP activity values (Fig. 6D), and the lowest values were registered for constructs kept under continuous flow perfusion. 


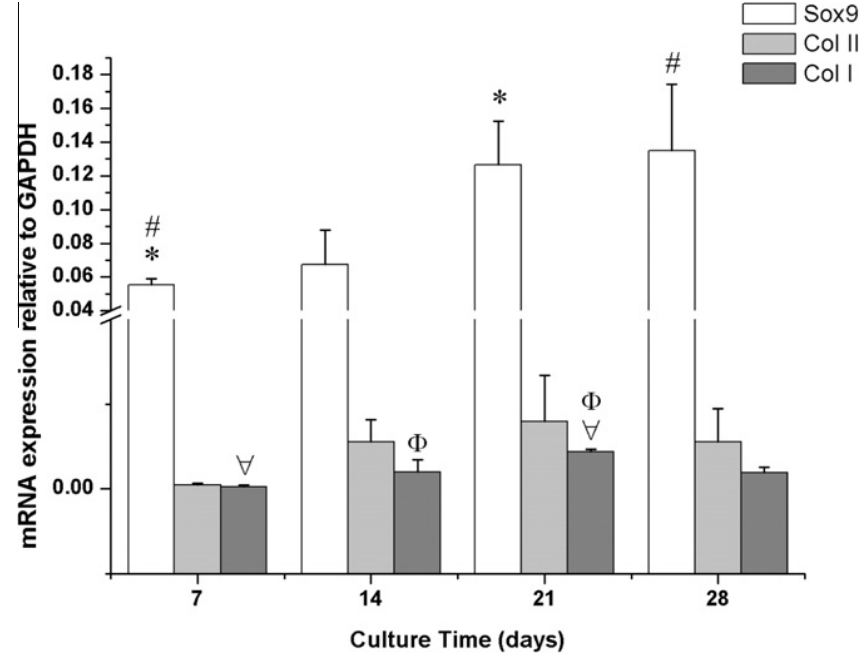

Fig. 5. mRNA expression of Sox9, Col II and Col I of pellets at different culture times, relative to the housekeeping GAPDH. Symbols indicate statistically significantly different $(P<0.05)$ expression levels at different time points.
SEM analysis showed that cells seeded onto the SPCL scaffolds were round in shape, a morphology typical of chondrocytes (Fig. 7A and B). This morphology was observed in both static and flow perfusion conditions. However, after 28 days in chondrogenic culture, cells started to modify their shape (data not shown).

To confirm the presence of PGs in the ECM, Safranin O staining was performed directly on the GBMC-SPCL constructs. All scaffolds used for Safranin O analysis are cell-seeded scaffolds, and Safranin $\mathrm{O}$ staining was homogeneously detected on the scaffold surface. The formation of ECM was detected in higher-magnification pictures by a darker red staining in some areas of the cultured scaffolds (Fig. 8).

After 4 weeks in chondrogenic medium culture supplemented with TGF- $\beta 1$, real-time RT-PCR was performed to evaluate the mRNA expression of Sox9, collagen IIA and collagen IA genes in the GBMC-SPCL constructs cultured under flow and static conditions. Sox9 is an important regulator of the chondrocytic phenotype and controls the expression of collagen IIA. The results are reported in Fig. 9. In general, cells cultured under different conditions showed different levels of gene expression. In particular, the flow perfusion culture seemed to enhance gene expression, and this is quite evident when comparing the expression of Sox9,
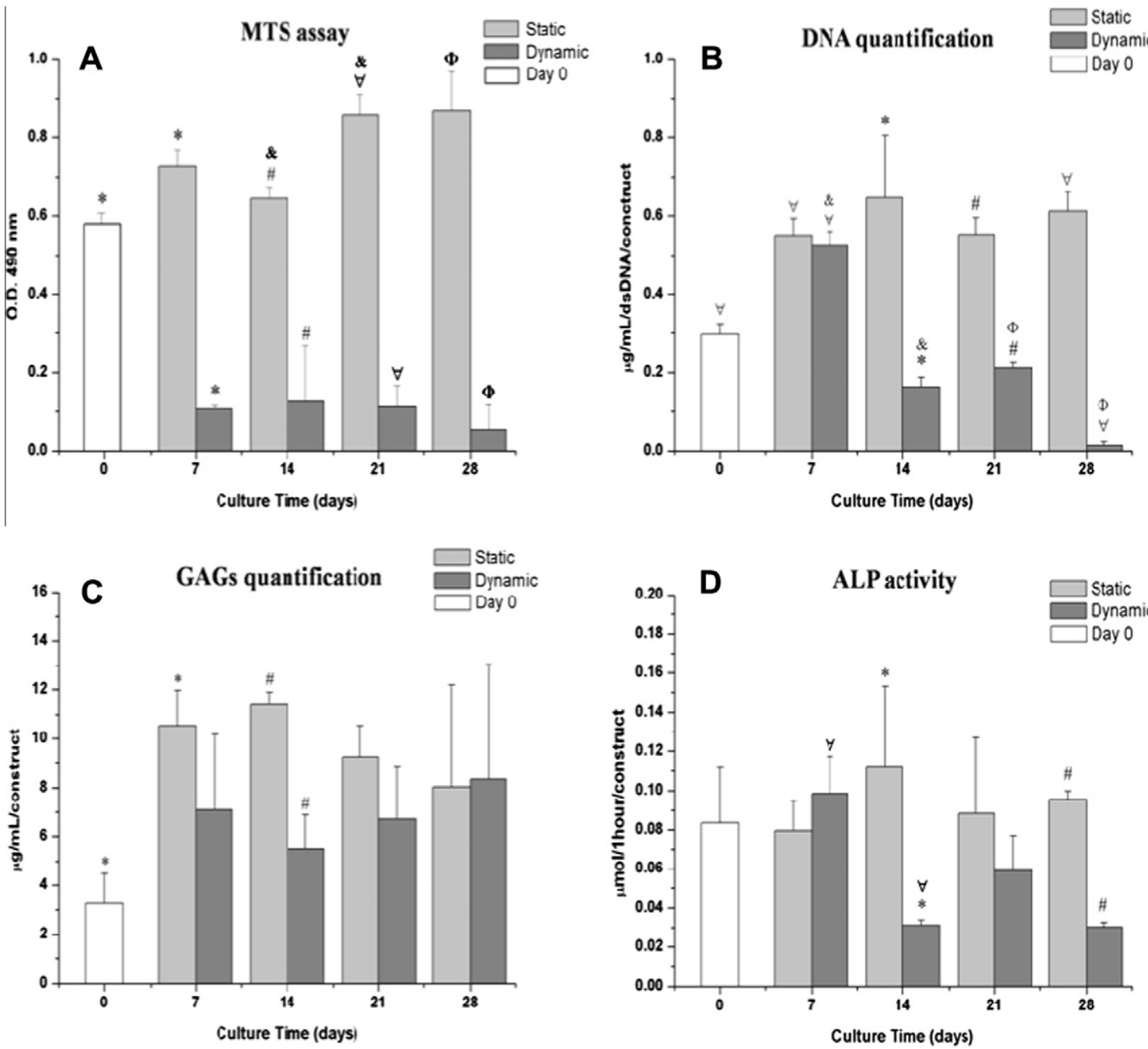

Fig. 6. GBMC-SPCL constructs cultured under chondrogenic medium in static and flow perfusion condition for 28 days: (A) cellular metabolic activity with MTS assay; (B) DNA content; (C) DMB assay for GAG quantification; (D) ALP activity. 

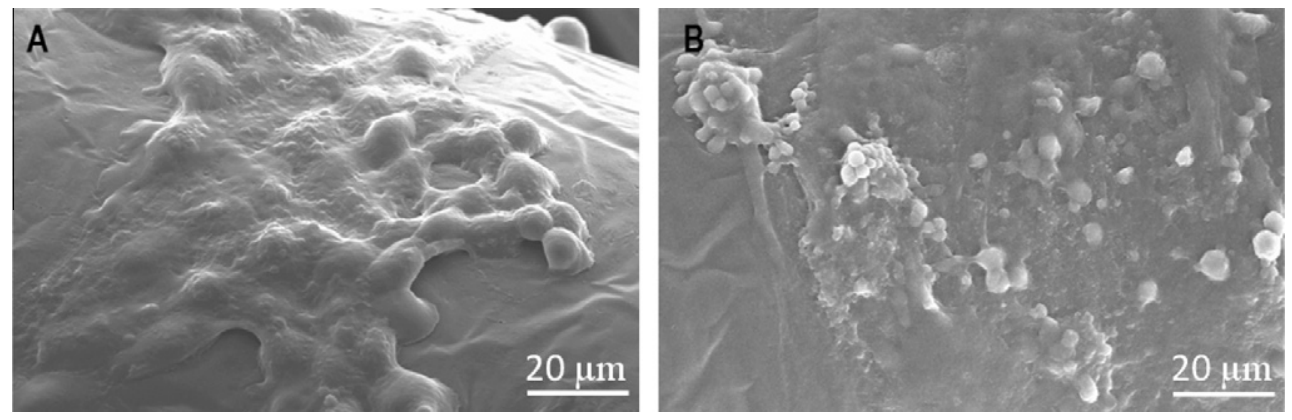

Fig. 7. SEM micrographs of the SPCL seeded with GBMCs and cultured in chondrogenic medium: (A) static condition; (B) flow perfusion condition.

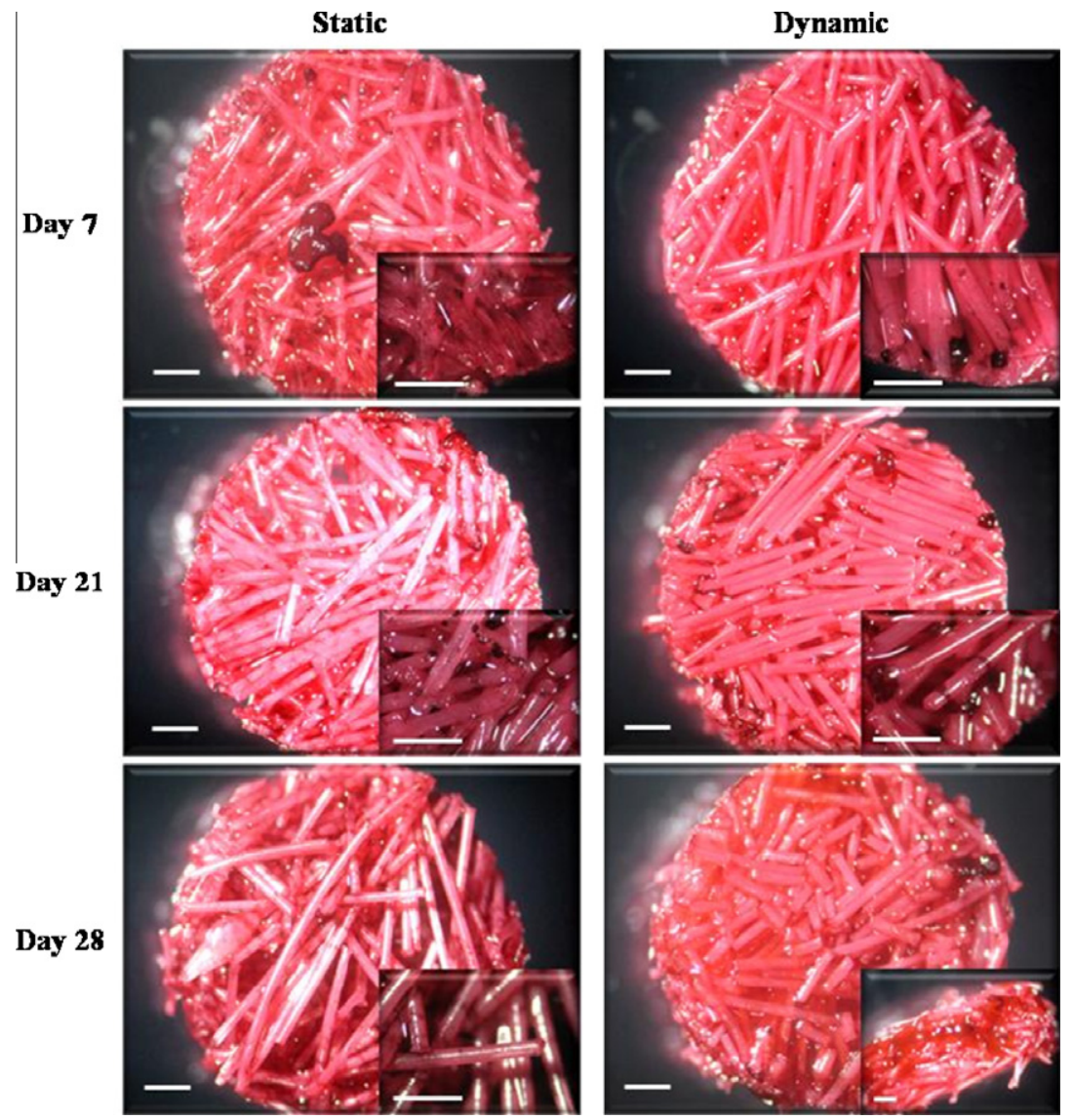

Fig. 8. Safranin O staining in SPCL-GBMC constructs. Scale bar $=1 \mathrm{~mm}$.

collagen IIA and collagen I to the values obtained for static culture. Higher expression of Sox9 is detected in an earlier stage of differentiation after 7 days in culture, while collagen IIA is highly expressed in the last culture end point (28 days).

\section{Discussion}

Mesenchymal progenitor cells with chondrogenic potential are present in many tissues of the body [10,12]. Those from bone marrow are of particular interest because of the ease of harvesting and their potential for autologous therapies promoting cartilage repair. Numerous studies have demonstrated that cells isolated from bone marrow can undergo in vitro osteogenic and/or chondrogenic differentiation under suitable culturing conditions $[1,3,14]$. The chondrogenic differentiation of MSCs from adult bone marrow involves a rapid biosynthesis of PGs and GAGs, the deposition of an inte- grated ECM, and is also accompanied by a dramatic alteration of cells into a round shaped morphology. This process is influenced by a number of key factors, including 3-D culture format, low oxygen tension and the presence of appropriate growth factors [22].

In this study, we first evaluated the ability of GBMCs to differentiate into the chondrogenic lineage through the pellet experiment. After the characterization of the chondrogenic differentiation process in GBMCs, we proceeded to the major goal of our study: the evaluation of the effect of flow perfusion in the chondrogenic differentiation of GBMCs seeded onto SPCL scaffolds.

\subsection{Pellet system culture}

This work describes the chondrogenic differentiation potential of mesenchymal progenitor cells from goat marrow, using SPCL scaffolds, to assemble cartilage-like tissue-engineered constructs 

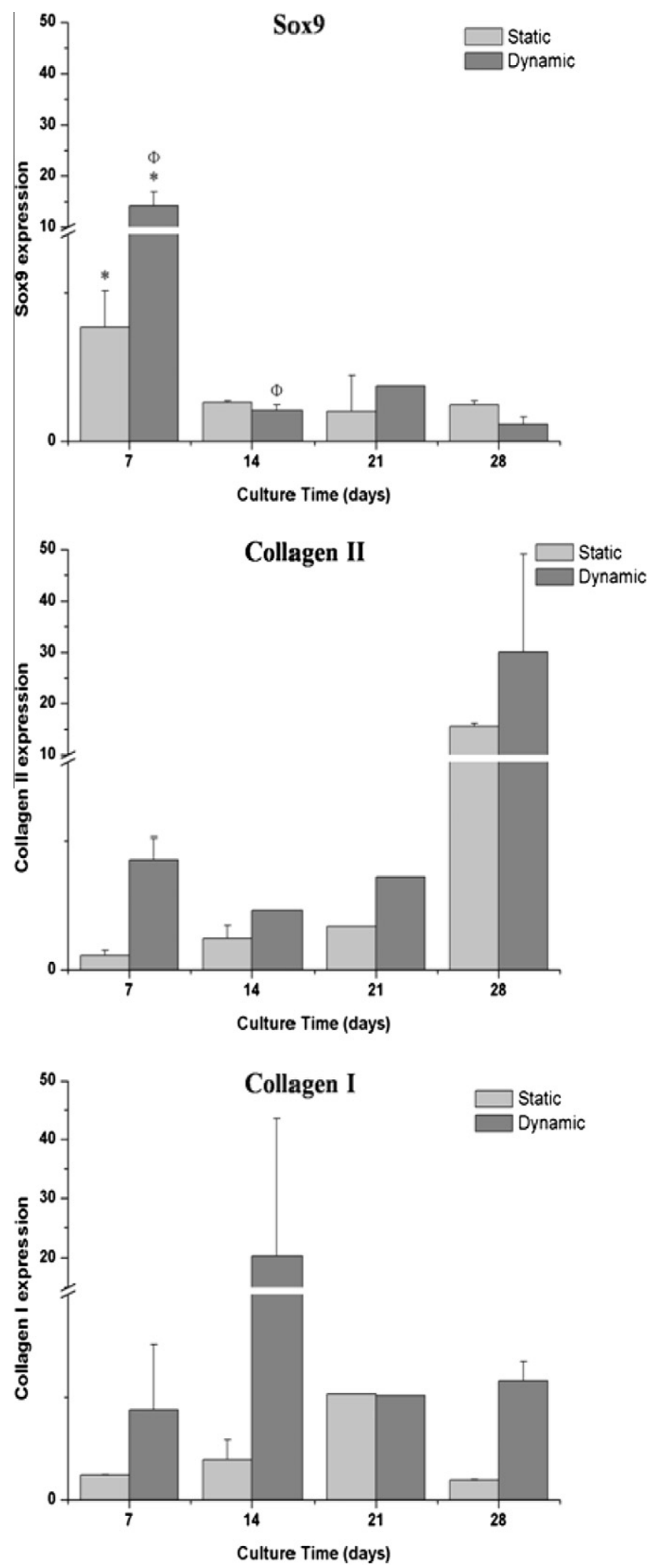

Fig. 9. Graphical representation of the results obtained from real-time RT-PCR analysis regarding the expression of different chondrogenic marker genes according to the culture condition.

under different culture conditions. GBMCs have been used in bone $[23,24]$, cartilage $[25,26]$ and also osteochondral $[6,27] \mathrm{TE}$.

In this study, TGF- $\beta 1$ and dexamethasone were used as factors to induce chondrogenic differentiation. TGF- $\beta 1$ provides a cellular stimulus for differentiation, regardless of the number of cells with chondrogenic capacity. In the pellet culture system, the addition of TGF- $\beta 1$ provided the appropriate external signal which, when coupled with endogenous factors, communicated between cells within the condensation, and facilitated chondrogenic differentiation $[22,28]$. The results on cell proliferation and metabolic activity rates (Figs. 1 and 2) showed that GBMCs delay the proliferation rate until day 28 , while they differentiate into the chondrogenic lineage, i.e. pellets display an initial phase of cell proliferation, followed by matrix deposition (Figs. 3 and 4). The positive staining areas for chondrocytic markers increased with culture time. After 28 days in culture almost all pellets are strongly stained for the different cartilage-specific stainings (Fig. 3A-H). Immunoreactivity for type IIA collagen also indicates that GBMCs undergo chondrogenic differentiation. Positive immunocytochemical staining was observed at all end points, but stronger staining areas were found after 28 days in chondrogenic medium, mainly located at the border layer of GBMC pellets (Fig. 4) [22]. Results from real-time RT-PCR confirmed the expression of three important and typical chondrogenic markers. Sox9 gene expression was up-regulated earlier in the flow culture compared to the static culture, which indicated an initial onset of chondrogenesis [29]. Gene expression of Sox9 observed on day 7 also suggested that GBMCs release signaling factors involved in the regulation of the chondrogenic differentiation.

In summary, the presence of Safranin O-, Toluidine blue- and Alcian blue-stained matrix, the chondrogenic morphology of cells, and the detection of type II collagen protein as well as Sox9 gene, clearly demonstrated the ability of goat marrow-derived cells to generate a "cartilage-like" tissue [22,30].

\subsection{GBMC-SPCL constructs in flow perfusion culture}

We next analyzed the chondrogenic potential of GBMCs combined with SPCL scaffolds cultured under flow perfusion or static conditions. In previous studies, SPCL fiber-mesh scaffolds have been shown [1] to support the proliferation and differentiation of bovine articular chondrocytes, demonstrating the potential of our study as a valid alternative strategy for cartilage TE.

Chondrocytes have been described as cells with low proliferative and migration rates but a high potential to form cellular aggregates. Considering that GBMCs have the potential to differentiate into the chondrogenic lineage with low movement potential, determined by the pellet experiment, this study was mainly focused on the effect of mechanical stimulation, promoted by the flow perfusion, to stimulate the chondrogenic differentiation of GBMCs.

Bioreactors are culture devices in which medium circulates dynamically, enhancing nutrient and metabolite transport, as well as mechanical stimulation, thus promoting tissue growth [31]. This system has been used to improve proliferation and differentiation of cells. Different bioreactors are used for different strategies and flow perfusion bioreactors are well known for inducing osteogenic differentiation [14]. An overview of the proliferation rate showed that during the first 7 days, DNA values are quite similar under both static and dynamic conditions, followed by a significant decrease in proliferation at week 2 in flow perfusion culture, while in static conditions DNA values are maintained through the experimental time (Fig. 6A and B). This can be associated with an earlier differentiation of GBMC-SPCL constructs under flow perfusion conditions. When cells differentiate, the proliferation rate decreases because a higher amount of cellular energy is taken by the synthesis of ECM matrix proteins rather than for cell replication and proliferation. However, the mechanism behind the direct perfusion on chondrogenic differentiation is not well understood and differences between reports may be related to the type of scaffolds or bioreactor systems used [32]. The main point, when comparing 
the results obtained in static and flow perfusion culture of GBMCSPCL constructs, is to analyze the evolution of the chondrogenic markers involved in this process.

SEM micrographs revealed that GBMCs seeded onto SPCL fibers had a round shaped morphology, typical of chondrocytic cells, under both static and flow perfusion conditions.

Concerning ECM production, GBMC-SPCL constructs stained Safranin $O$ throughout the scaffold; in higher-magnification pictures, darker areas correlate to a higher amount of ECM.

After 4 weeks in culture, the analysis of gene expression indicated that different stages of chondrogenic differentiation are distinguished in both cultures. However, it is important to highlight that, in general, the flow perfusion culture showed higher values of gene expression for all analyzed genes (Fig. 9). In an initial stage, Sox9 mRNA is overexpressed at day 7 in both cultures (Fig. 9, Sox9). Sox9 is a master transcriptional factor (TF) required for chondrocyte differentiation [33]. This nuclear TF is an early marker expressed in cells undergoing differentiation, and is required for the gene expression of type IIA collagen (Col2a1) and other cartilage-specific matrix proteins [34]. Collagen II is a well-known marker of cartilage ECM, usually expressed at the final stage of the chondrogenic differentiation process. Furthermore, Sox9 might also control the expression of genes encoding cell surface proteins desirable for mesenchymal condensation [33]. Our results showed a higher value of type IIA collagen gene expression at day 7 when compared to day 14 in static culture (Fig 9, Collagen IIA). Unlike type IIA collagen, type I (Col1a1) collagen expression increased between 7 and 14 days of culture. At this stage, cells could be considered as pre-chondrogenic cells [34]. Prior to condensation, prechondrocyte cells produce hyaluronic and type I collagen-rich ECM, as well as type IIA collagen. The amount of type II pro-collagen is controlled at the molecular level by the transcription of mRNA from Col2a1 gene. In addition, type II collagen can be expressed in two isoforms by differential splicing of the primary gene transcript, using a post-transcription control mechanism. Since type II collagen alpha 1 expression is up-regulated, a different splice variant of the gene, the type IIB, might be expressed instead of type IIA $[34,35]$. The variant of type II collagen being expressed was not identified, but could explain the decrease in Col IIA mRNA values detected in both cultures.

In the final culture end point, day 28, GBMCs might have emerged into a chondrocyte-like stage, supported by the fact that, at this level, mRNA expression of Sox9 is turning off and mRNA levels of type IIA collagen increased, when compared to other culturing times [35].

ALP was included in this study as a hypertrophy marker to detect if GBMCs were differentiating into hypertrophic chondrocytes at a later stage of the differentiation process. Hypertrophic chondrocytes are characterized by a simultaneous increase in ALP and type I collagen levels. Although at the 2 week end point, an increase in the expression of these two markers is observed, related to cellular condensation, hypertrophy is described to occur only after 4 weeks in chondrogenic culture [35].

\section{Conclusions}

In this study SPCL scaffolds seeded with GBMCs in a chondrogenic medium supplemented with TGF- $\beta 1$ showed enhanced mRNA expression for chondrogenic genes and enhanced production of ECM when cultured under dynamic (flow perfusion) conditions. The production of ECM is crucial for the chondrogenic differentiation process, because ECM is the main tissue support. In this sense, larger amounts of ECM produced in vitro are likely to promote better results after in vivo implantation. In conclusion, the flow perfusion system used in this work seemed to create a favorable environment to promote the chondrogenic differentiation of GBMCs cultured onto SPCL fiber meshes, and therefore with the potential to produce in vitro highly functional constructs for further in vivo implantation.

\section{Acknowledgements}

This work was partially supported by the Portuguese Foundation for Science and Technology (FCT) Project VivoTissue (PTDC/ CVT/67677/2006) and was carried out under the scope of the European NoE EXPERTISSUES (NMP3-CT-2004-500283).

\section{Appendix A. Figures with essential colour discrimination}

Certain figures in this article, particularly Figures 3, 4 and 8, are difficult to interpret in black and white. The full colour images can be found in the on-line version, at doi:10.1016/j.actbio.2010. 11.044 .

\section{References}

[1] Oliveira JT. A cartilage tissue engineering approach combining starchpolycaprolactone fibre mesh scaffolds with bovine articular chondrocytes. J Mater Sci-Mater Med 2007;18:295-302.

[2] Ahmed N. Cartilage tissue formation using redifferentiated passaged chondrocytes in vitro. Tissue Eng A 2009;15:665-73.

[3] da Silva MA. Evaluation of extracellular matrix formation in polycaprolactone and starch-compounded polycaprolactone nanofiber meshes when seeded with bovine articular chondrocytes. Tissue Eng A 2009;15:377-85.

[4] Schulz RM. Cartilage tissue engineering and bioreactor systems for the cultivation and stimulation of chondrocytes. Eur Biophys J 2007;36:539-68.

[5] Cancedda R. Tissue engineering and cell therapy of cartilage and bone. Matrix Biol 2003;22:81-91.

[6] Oliveira JM. Novel hydroxyapatite/chitosan bilayered scaffold for osteochondral tissue-engineering applications: scaffold design and its performance when seeded with goat bone marrow stromal cells. Biomaterials 2006;27:6123-37.

[7] Hardingham T. Tissue engineering: chondrocytes and cartilage. Arthritis Res 2002;4(Suppl. 3):S63-8.

[8] Chung C. Engineering cartilage tissue. Adv Drug Deliv Rev 2008:60:243-62.

[9] Marolt D. Bone and cartilage tissue constructs grown using human bone marrow stromal cells, silk scaffolds and rotating bioreactors. Biomaterials 2006;27:6138-49.

[10] Heng BC. Directing stem cell differentiation into the chondrogenic lineage in vitro. Stem Cells 2004;22:1152-67.

[11] Salgado AJ. Adult stem cells in bone and cartilage tissue engineering. Curr Stem Cell Res Ther 2006;1:345-64.

[12] Robey PG. The use of adult stem cells in rebuilding the human face. J Am Dent Assoc 2006;137:961-72.

[13] Pazzano D. Comparison of chondrogensis in static and perfused bioreactor culture. Biotechnol Prog 2000;16:893-6.

[14] Gomes ME. Effect of flow perfusion on the osteogenic differentiation of bone marrow stromal cells cultured on starch-based three-dimensional scaffolds. J Biomed Mater Res A 2003;67:87-95.

[15] Gomes ME. Tissue engineering: key elements and some trends. Macromol Biosci 2004;4:737-42.

[16] Martin I. The role of bioreactors in tissue engineering. Trends Biotechnol 2004;22:80-6.

[17] Gomes ME. Influence of the porosity of starch-based fiber mesh scaffolds on the proliferation and osteogenic differentiation of bone marrow stromal cells cultured in a flow perfusion bioreactor. Tissue Eng 2006;12:801-9.

[18] Gomes ME. Starch-poly(epsilon-caprolactone) and starch-poly(lactic acid) fibre-mesh scaffolds for bone tissue engineering applications: structure, mechanical properties and degradation behaviour. J Tissue Eng Regen Med 2008;2:243-52.

[19] Costa PF, Martins A, Neves NM, Gomes ME, Reis RL. A novel bioreactor design for enhance stem cells proliferation in tissue engineered constructs. Tissue Eng A 2008;14.

[20] Pedro Costa AM, Nuno M. Neves, Manuela Gomes, Rui L. Reis. Multichamber bioreactor with bidirectional perfusion integrated in culture system for tissue engineering strategies. European Patent 09009863. 3-1521, 2009.

[21] Livak KJ. Analysis of relative gene expression data using real-time quantitative PCR and the $2^{-\Delta \Delta \mathrm{Ct}}$ method. Methods 2001;25:402-8.

[22] Bosnakovski D. Chondrogenic differentiation of bovine bone marrow mesenchymal stem cells in pellet cultural system. Exp Hematol 2004;32:502-9.

[23] Ivirico JL. Proliferation and differentiation of goat bone marrow stromal cells in 3D scaffolds with tunable hydrophilicity. J Biomed Mater Res B Appl Biomater 2009;91:277-86. 
[24] Kruyt MC. Optimization of bone-tissue engineering in goats. J Biomed Mater Res B Appl Biomater 2004;69:113-20.

[25] Klein-Nulend J. Osteogenic protein (OP-1, BMP-7) stimulates cartilage differentiation of human and goat perichondrium tissue in vitro. J Biomed Mater Res 1998;40:614-20.

[26] Miot S. Cartilage tissue engineering by expanded goat articular chondrocytes. Orthop Res 2006;24:1078-85.

[27] Murphy JM. Stem cell therapy in a caprine model of osteoarthritis. Arthritis Rheum 2003;48:3464-74.

[28] Johnstone B. In vitro chondrogenesis of bone marrow-derived mesenchyma progenitor cells. Exp Cell Res 1998;238:265-72.

[29] Ahmed N. Soluble signalling factors derived from differentiated cartilage tissue affect chondrogenic differentiation of rat adult marrow stromal cells. Cell Physiol Biochem 2007;20:665-78.
[30] Barry F. Chondrogenic differentiation of mesenchymal stem cells from bone marrow: differentiation-dependent gene expression of matrix components. Exp Cell Res 2001;268:189-200.

[31] Sikavitsas VI. Flow perfusion enhances the calcified matrix deposition of marrow stromal cells in biodegradable nonwoven fiber mesh scaffolds. Ann Biomed Eng 2005;33:63-70.

[32] Schulz RM. Development and validation of a novel bioreactor system for loadand perfusion-controlled tissue engineering of chondrocyte-constructs. Biotechnol Bioeng 2008;101:714-28.

[33] de Crombrugghe B. Transcriptional mechanisms of chondrocyte differentiation. Matrix Biol 2000:19:389-94.

[34] Goldring MB. The control of chondrogenesis. J Cell Biochem 2006;97:33-44.

[35] Lefebvre V. Transcriptional control of chondrocyte fate and differentiation. Birth Defects Res C Embryo Today 2005;75:200-12. 